\title{
A simple prognostic index based on admission vital signs data among patients with sepsis in a resource-limited setting
}

\author{
Stephen B Asiimwe ${ }^{1,3^{*}}$, Amir Abdallah ${ }^{1,2}$ and Richard Ssekitoleko ${ }^{4}$
}

\begin{abstract}
Introduction: In sub-Saharan Africa, vital signs are a feasible option for monitoring critically ill patients. We assessed how admission vital signs data predict in-hospital mortality among patients with sepsis. In particular, we assessed whether vital signs data can be incorporated into a prognostic index with reduced segmentation in the values of included variables.
\end{abstract}

Methods: Subjects were patients with sepsis hospitalized in Uganda, who participated in two cohort studies. Using restricted cubic splines of admission vital signs data, we predicted probability of in-hospital death in the development cohort and used this information to construct a simple prognostic index. We assessed the performance of the index in a validation cohort and compared its performance to that of the Modified Early Warning Score (MEWS).

Results: We included 317 patients (167 in the development cohort and 150 in the validation cohort). Based on how vital signs predicted mortality, we created a prognostic index giving a score of 1 for: respiratory rates $\geq 30$ cycles/minute; pulse rates $\geq 100$ beats/minute; mean arterial pressures $\geq 110 /<70 \mathrm{mmHg}$; temperatures $\geq 38.6 /<35.6^{\circ} \mathrm{C}$; and presence of altered mental state defined as Glasgow coma score $\leq 14 ; 0$ for all other values. The proposed index ( maximum score $=5$ ) predicted mortality comparably to MEWS. Patients scoring $\geq 3$ on the index were 3.4-fold (95\% confidence interval $(\mathrm{Cl}) 1.6$ to $7.3, P=0.001)$ and 2.3 -fold ( $95 \% \mathrm{Cl} 1.1$ to $4.7, P=0.031$ ) as likely to die in hospital as those scoring 0 to 2 in the development and validation cohorts respectively; those scoring $\geq 5$ on MEWS were 2.5 -fold ( $95 \% \mathrm{Cl} 1.2$ to $5.3, P=0.017$ ) and 1.8 -fold ( $95 \% \mathrm{Cl} 0.74$ to $4.2, P=0.204$ ) as likely to die as those scoring 0 to 4 in the development and validation cohorts respectively.

Conclusion: Among patients with sepsis, a prognostic index incorporating admission vital signs data with reduced segmentation in the values of included variables adequately predicted mortality. Such an index may be more easily implemented when triaging acutely-ill patients. Future studies using a similar approach may develop indexes that can be used to monitor treatment among acutely-ill patients, especially in resource-limited settings.

\section{Introduction}

In sub-Saharan Africa (SSA), sepsis is common and is associated with high mortality [1]. Also, available treatment interventions and tools to guide their administration are few. Although studies are increasingly being performed in the region to test various interventions,

\footnotetext{
* Correspondence: asiimwesteve@gmail.com

'Department of Medicine, Mbarara Regional Referral Hospital, P.O. Box 40, Mbarara, Uganda

${ }^{3}$ Department of Epidemiology and Biostatistics, University of California San Francisco, 550 16th Street, Second Floor, Mission Hall: Global Health and Clinical Sciences Building, San Francisco, CA 94158-2549, USA

Full list of author information is available at the end of the article
}

the design of such studies is affected by lack of monitoring tools [2].

In resource-rich environments, vital parameters such as the central venous pressure (CVP), mean arterial pressure (MAP), and central venous oxygen saturation ( $\mathrm{ScvO} 2)$ commonly guide treatment of patients with sepsis [3]. Initial values of these variables and subsequent changes thereof are considered when deciding whether or not to give, escalate, or de-escalate interventions such as intravenous fluids, diuretics, supplemental oxygen, blood transfusion, and vasopressors or, if needed, vasodilators [4]. As these variables are commonly measured using invasive techniques, and also their prognostic 
properties are not fully understood, their use in guiding treatment continues to be challenged [5,6]. Additionally, it is difficult to use such variables to monitor treatment in resource-limited settings, where monitoring facilities are scarce.

Better tools to monitor treatment of patients with sepsis are therefore required in all settings. However, this need is more acute in SSA, where any mildly sophisticated monitoring tools are rarely available, and critically-ill patients are treated from general wards $[7,8]$. In this environment, routine vital signs are among the feasible options to monitor treatment. Routine vital signs are particularly appealing for this purpose; they represent a treatment-modifiable signal that can be obtained using cheaper and non-invasive techniques. However, their prognostic utility has not been adequately studied.

A number of existing prognostic indexes incorporate vital signs data [9-11]. As these indexes predict posthospitalization mortality [10-12], they can be used to triage acutely-ill patients, which is how they are predominantly used in resource-rich settings [13]. Such indexes could also be used to monitor treatment, especially in resource-limited settings like SSA, if their prognostic properties were adequate, which is not the case [14]. Although they predict mortality $[10,15]$, their accuracy and efficiency remain inadequate [16], and indexes like the Modified Early Warning Score (MEWS), where the values of included variables are heavily segmented $[17,18]$, can be difficult to implement in routine clinical settings. It is not clear if the segmentation in the MEWS can be reduced while preserving prognostic properties.

Recent studies in SSA have focused on validating lesssegmented prognostic indexes. In a recent study in Malawi, two alternative indexes; one incorporating hypotension, oxygen saturation, temperature, electrocardiographic abnormality, and loss of independence (HOTEL); and another, incorporating tachypnea, oxygen saturation, temperature, alertness, and loss of independence (TOTAL), performed better than MEWS in predicting mortality [14]. However, these indexes also contain variables that are not always available, for example the peripheral oxygen saturation, and variables whose prognostic abilities are not adequately understood, for example loss of independence, and include some variables in ways that may not be efficient, for example including both tachypnea and oxygen saturation.

Among patients hospitalized with sepsis, we aimed to describe how admission vital signs data predict mortality. We hoped to use this information to create a prognostic index incorporating vital signs data with less segmentation in the values of included variables. Improved prognostic indexes that use vital signs data may be used to monitor treatment of patients with sepsis.

\section{Methods}

We analyzed data of adults hospitalized with sepsis, who were enrolled in two cohort studies at the Mbarara Regional Referral Hospital in Mbarara, Uganda. Using admission vital signs data in one cohort (the development cohort), we constructed a prognostic index to predict mortality. To determine how the index would perform in a different patient group, we assessed its performance in another cohort (the validation cohort). The setting and study populations of the two cohorts have been described elsewhere $[8,19]$. Briefly, the first study occurred in April to June 2011 and prospectively enrolled adults hospitalized for any medical illness [19]. From the patients enrolled in this study $(n=318)$, we selected those meeting criteria for sepsis at admission $(n=167)$, who comprised the development cohort. All patients enrolled in this cohort provided written informed consent to participate. The second study occurred in February to July 2009 and had prospectively enrolled patients meeting criteria for sepsis $(n=150)$ [8], who comprised the validation cohort. These patients also had provided written informed consent to participate. Both studies were approved by the Institutional Review Committee (IRC) at Mbarara University of Science and Technology.

\section{Measurements}

Study definitions and procedures for measuring vital signs were similar across the two cohorts. We defined sepsis and severe sepsis using clinical definitions, since definitions based on complex laboratory testing would be difficult to implement in this setting. Accordingly, sepsis was defined as the presence of suspected infection, plus two or more of the systemic inflammatory response syndrome (SIRS) criteria (pulse $\geq 90$ beats/minute; respiratory rate $\geq 20$ cycles/minute; a temperature $\geq 38^{\circ} \mathrm{C}$ or $\leq 36^{\circ} \mathrm{C}$; and white blood cell count $\geq 12,000$ cells/cc, or $<4,000$ cells/cc, or $>10 \%$ band forms). Severe sepsis was defined as the presence of sepsis plus at least one organ dysfunction (Glasgow coma score (GCS) $<15$, systolic blood pressure (SBP) $<90 \mathrm{mmHg}$, or platelet counts $<100,000$ cells/cc) [20]. Blood pressures were measured using standard techniques and a manual cuff; all temperatures were axillary, and pulse and respiratory rates were obtained by manual counting. We calculated the MAP as a weighted average of the SBP and the diastolic BP (DBP) (SBP plus twice the DBP divided by 3) [21] and assessed mental state using the GCS. For all variables, the admission value was the first measurement obtained from the patient on their day of admission.

\section{Determination of in-hospital mortality}

In both cohorts, patients were followed in hospital until death or discharge, and there were no losses to follow-up. 


\section{Analysis}

\section{Descriptive summaries}

We first summarized patient characteristics in each cohort using medians and inter-quartile ranges (IQR) for vital signs, as well as appropriate summaries for age, sex, severity of sepsis, mental state, and suspected focus of infection. We proceeded to assess relationships between vital signs and mortality and to develop and validate the proposed prognostic index as described below.

\section{Development of prediction rules and the prognostic index}

In the development cohort, we used restricted cubic splines of admission vital signs data to predict the probability of in-hospital death; restricted cubic splines are a flexible way of assessing relationships [22-24]. Based on the shapes of smoothed curves of vital signs data against probability of in-hospital death, we created prognostic and reference categories in the values of each vital sign. We defined reference categories as those with low mortality; that is below or about the sample average (23\%). Prognostic categories were those where mortality was above this average. We then calculated average mortality in each category. Using this information, we determined cutoffs in the values of each vital sign below or above which a score of 1 would be given in a simple scoring system to create a prognostic index. For mental state data, we classified patients by GCS level as: $\leq 12,13$ to 14 , and 15. Guided by the distribution of mortality in these categories, we also created a binary cutoff for GCS. We assessed the performance of the proposed index in the development cohort by calculating proportions dying at different scores, as well as unadjusted odds ratios (OR) and $95 \%$ confidence intervals (CI) comparing mortality in subjects with scores $\geq 3$ to mortality in those with scores of 0 to 2 .

\section{Validation of prediction rules and the proposed index}

To better understand whether the prediction patterns observed in the development cohort could be replicated in a different patient group, we created similar reference and prognostic categories in the values of each vital sign in the validation cohort. Further, we assessed the performance of the proposed index in the validation cohort in the same way that we assessed its performance in the development cohort (that is, by calculating proportions dying at different scores, as well as unadjusted ORs and 95\% CI comparing mortality in subjects with scores of $\geq 3$ to mortality in those with scores of 0 to 2 ). In both cohorts, we also compared the performance of the proposed index to that of the MEWS.

\section{Statistical issues}

In the development cohort, we imputed 11 to 34 missing values on respiratory rate, white cell counts, and GCS using multiple imputation (MI) techniques and the other variables with complete data $[25,26]$. We then used 20 extracted and averaged post-MI datasets to perform all analyses of the development cohort. The medians and IQRs for the respiratory rate, white cell counts, and GCS in this cohort, as well as the restricted cubic spline models, were therefore obtained from post-MI datasets. There were no missing data in the validation cohort.

\section{Results}

\section{Subject characteristics}

\section{Development cohort}

We included in the development cohort 167 patients with sepsis (61\% had severe sepsis) hospitalized in April to June 2011. Median age was 38 (IQR 28 to 55). Fifty-three percent were male, and the largest proportion (60\%) had suspected chest infection. In this cohort, in-hospital mortality was $23 \%$ overall, and $29 \%$ in those with severe sepsis. Overall median length of stay in hospital was 6 days (IQR 6 to 10$)$.

\section{Validation cohort}

This cohort comprised 150 patients with sepsis (65\% had severe sepsis) hospitalized in February to July 2009. Median age was 31 (IQR 25 to 41). Sixty-three percent were male, and the largest proportion also had suspected chest infection (64\%) (Table 1). In-hospital mortality was $30 \%$ overall, and $41 \%$ in those with severe sepsis. Overall length of stay in hospital was 4 days (IQR 1 to 7).

\section{Relationships of admission vital signs data with mortality} Admission MAP and temperature predicted mortality with approximately U-shaped relationships; pulse and respiratory rates predicted mortality in roughly linear relationships (Figure 1). Altered mental state also predicted mortality; mortality was $40 \%, 33 \%$, and $20 \%$ in patients with GCS $\leq 12,13$ to 14 , and 15 , respectively. Consequently, we determined that the prognostic values for the GCS variable would be $\leq 14$; patients with GCS $\leq 14$ were 2.4 -fold ( $95 \%$ CI 1.1 to $5.4, P=0.033$ ) as likely to die as those with GCS of 15 .

\section{Prediction rules for constructing the proposed prognostic index}

Using the shapes of the graphs (Figure 1) and how altered mental state predicted mortality, we determined the following rules: first, that a scoring system giving a score of 1 for high respiratory rate ( $\geq 30$ cycles/minute), high pulse rate ( $\geq 100$ beats/minute), high or low MAP $(\geq 110$ or $<70$ $\mathrm{mmHg})$, high or low temperature $\left(\geq 38.6^{\circ} \mathrm{C}\right.$ and $\left.<35.6\right)$, and altered mental state (GCS $\leq 14)$, and 0 for all other values (Table 2), would adequately predict mortality; second, that in such an index, low values of respiratory and pulse rates may also be given a score of 0 . 
Table 1 Characteristics at admission of 317 patients with sepsis hospitalized in 2009 and 2011 in Uganda

\begin{tabular}{lll}
\hline Variable & $\begin{array}{l}\text { Development } \\
\text { cohort }(n=167)\end{array}$ & $\begin{array}{l}\text { Validation } \\
\text { cohort }(n=150)\end{array}$ \\
\hline
\end{tabular}

Numeric variables, median (IQR)

Age

Pulse (beats/minute)

108 (90 to120) $110(104$ to 120$)$

Temperature $\left({ }^{\circ} \mathrm{C}\right)$

37 (36.5 to 38.3 ) $\quad 38.5$ (38 to 39 )

Respiratory rate (cycles/minute)

28 (22 to 32 )

30 (24 to 36$)$

Systolic blood pressure $(\mathrm{mmHg})$

100 (90 to 120$)$

110 (85 to 110$)$

Diastolic blood pressure $(\mathrm{mmHg})$

60 (50 to 70$)$

60 (50 to 70$)$

Mean arterial pressure $(\mathrm{mmHg})$

73 (63 to 87 )

White cell counts $\left(\times 10^{3}\right.$ cells/cc)

Platelets $\left(\times 10^{3}\right.$ cells/cc)

4.7 (2.7 to 6.2)

73 (63 to 88)

160 (108 to 229)

(3.2 to 9.2)

Binary variables, n (\%)

Sex male

$88(53 \%)$

$94(63 \%)$

Altered mental state

$35(21 \%)$

$35(23 \%)$

Severe sepsis

$101(61 \%)$

97 (65\%)

Suspected focus of infection

Chest

Central nervous system

$100(60 \%)$

96 (64\%)

Gastro-intestinal

45 (27\%)

$18(12 \%)$

$2(1.2 \%)$

$17(11 \%)$

Other focus

20 (12\%)

19 (13\%)

The table shows the characteristics of patients admitted with sepsis at Mbarara Regional Referral Hospital in Uganda. One hundred and sixty-seven patients hospitalized in April to June 2011 were included in the development cohort, and 150 patients with sepsis hospitalized in February to July 2009 were included in the validation cohort. IQR, inter-quartile range.

\section{Validation of the proposed prediction rules}

In assessing whether the proposed prediction rules were reliable, patterns of mortality in the prognostic categories of different vital signs were roughly similar across the two cohorts (Table 3).

\section{Prediction of mortality by the proposed index}

The prognostic index (total possible score $=5$ ) developed according to the above rules (Table 2 ) adequately predicted mortality in the development and validation cohorts; mortality increased with increasing scores of the proposed index. For example, mortality was $11 \%$ at a score of 0 , increasing to $25 \%$ at a score of 2 and to $36 \%$ at score of 3 in the development cohort; mortality was $27 \%$ and $16 \%$ at scores of 1 and 2 respectively, increasing to $34 \%$ at a score of 3 in the validation cohort (Table 4 ).

\section{Performance of the proposed index and MEWS in the development cohort}

The proposed prognostic index performed well and comparably to MEWS in the development cohort; patients scoring $\geq 3$ on the proposed index were 3.4 -fold (95\% CI
1.6 to $7.3, P=0.001$ ) as likely to die in hospital as those scoring 0 to 2 ; those scoring $\geq 5$ on MEWS were 2.5-fold (95\% CI 1.2 to $5.3, P=0.017$ ) as likely to die as those scoring 0 to 4 (Table 5).

\section{Performance of the proposed index and MEWS in the validation cohort}

The proposed index also performed well and comparably to MEWS in the validation cohort; patients scoring $\geq 3$ on the proposed index were 2.3 -fold (95\% CI 1.1 to 4.7 , $P=0.031)$ as likely to die as those scoring 0 to 2 ; those scoring $\geq 5$ on MEWS were 1.8 -fold ( $95 \%$ CI 0.74 to 4.2 , $P=0.204$ ) as likely to die as those scoring 0 to 4 (Table 5).

\section{Discussion}

In SSA, routine vital signs are among the feasible options to monitor treatment of critically ill patients. Among patients hospitalized with sepsis in Uganda, we used restricted cubic splines and plotted smoothed curves of admission vital signs data against the probability of in-hospital death. Using this information, we determined rules to create a composite prognostic index incorporating vital signs data. Our findings suggest that prognostic indexes may include vital signs data with reduced segmentation in the values of included variables, and that low values of respiratory and pulse rates may not be scored. A proposed prognostic index based on these rules and following a similar principle as MEWS [18] but with less segmentation adequately predicted mortality and compared favorably to MEWS.

Prognostic indexes using vital signs data usually contain substantial segmentation in the values of incorporated variables. For example, MEWS gives: 3 points for each of SBP $<70 \mathrm{mmHg}$, pulse rate $\geq 130$ beats/minute, respiratory rate $\geq 30$ cycles/minute, and GCS score $\leq 8 ; 2$ points for each of SBP 70 to 80 or $\geq 200 \mathrm{mmHg}$, pulse 111 to 129 beats/minute, respiratory rate $<9$ or 21 to 29 cycles/ minute, temperature $<35$ or $\geq 38.5^{\circ} \mathrm{C}$, and GCS 9 to 13 ; 1 point each for SBP 80 to $100 \mathrm{mmHg}$, pulse rate 40 to 50 or 101 to 110 beats/minute, respiratory rate 16 to 20 cycles/ minute, and GCS score of 14; and 0 for all other values [18]. A recent modification suggested for use in SSA includes more variables (seven in total) and more segmentation in some variables [17]. The high degree of segmentation may affect the efficiency and reliability of these indexes [27] and, in routine clinical settings, their applicability.

Our data suggest that less segmentation may be achieved in at least four vital signs. In blood pressure (BP) and temperature, average mortality on either side of the reference categories did not differ substantially. Although extreme values may be associated with higher mortality than intermediate values, the data in the extreme ranges are also likely to be thin most of the time. 

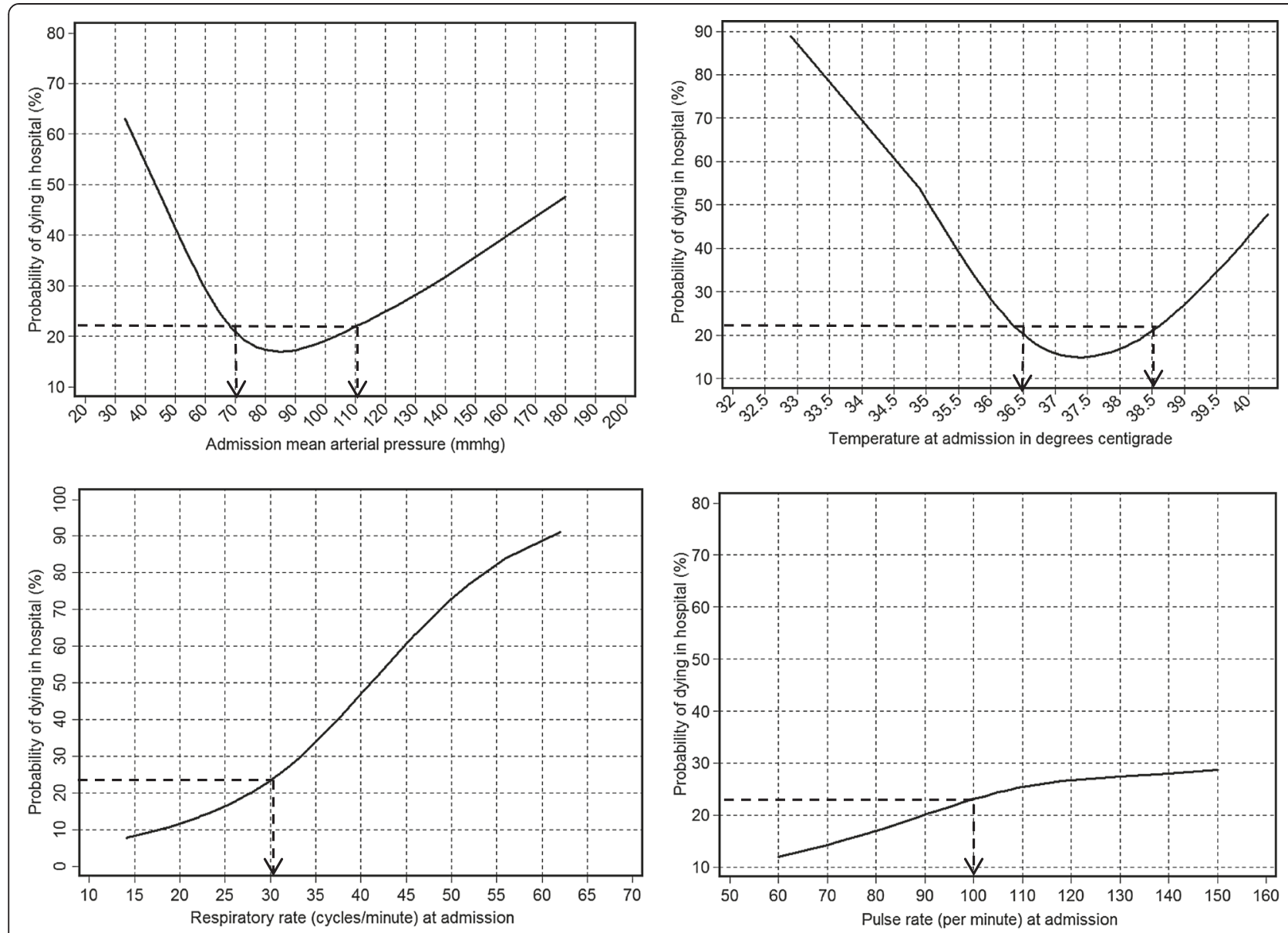

Figure 1 Probability of in-hospital death by admission mean arterial pressure, temperature, and respiratory and pulse rates. The figure shows smoothed predictions for the probability of in-hospital death according to the admission mean arterial pressure (MAP), temperature, and respiratory and pulse rates among 167 patients hospitalized with sepsis in April to June 2011 in Uganda. Admission MAP and temperature predicted mortality with approximately U-shaped relationships; mortality increased as either variable increased or decreased starting from a reference range (70 to $109 \mathrm{mmHg}$ for MAP and 36.6 to $38.5^{\circ} \mathrm{C}$ for temperature). Admission respiratory and pulse rates predicted mortality in roughly linear relationships; at respiratory rates $\geq 30$ cycles/minute mortality appeared to increase approximately linearly as respiratory rate increased. For the pulse rate, mortality did not go above average until a pulse rate $\geq 100$ beats/minute and tapered off close to the average mortality for this population (about 23\%) as the pulse rate increased. Using these patterns, we determined cutoffs (shown by arrows on the figure) below or above which we gave a score of 1 in the proposed prognostic index.

Table 2 Abnormalities in vital signs to receive a score of 1 in a proposed prognostic index

\begin{tabular}{lll}
\hline Vital sign & Abnormality & Cutoff $^{*}$ \\
\hline Respiratory rate (cycles/minute) & High & $\geq 30$ \\
Mean arterial pressure & High or low & $\geq 110$ or $<70$ \\
Temperature $\left({ }^{\circ} \mathrm{C}\right)$ & High or low & $\geq 38.6$ or $<35.6$ \\
Pulse rate (beats/minute) & High & $\geq 100$ \\
Altered mental state/GCS & Low GCS & GCS $\leq 14$ \\
\hline
\end{tabular}

"The proposed index gives a score of 1 for the indicated ranges and a score of 0 for all other values. The cutoffs are based on how each of the vital signs predicted mortality in the development cohort. GCS, Glasgow coma score.
For respiratory rate and pulse, mortality did not go above average until respiratory rate $\geq 30$ cycles/minute and pulse rate $\geq 100$ beats/minute respectively. In addition, low respiratory rates were rare (in both cohorts, no patient had respiratory rate $<12$ cycles/minute). We interpret these patterns to mean that in composite prognostic indexes using vital signs data, low and high values of BP and temperature may be scored similarly, and that for pulse and respiratory rate, scoring only the high values may be adequate.

Many prognostic scoring systems have been developed for monitoring critically-ill patients. These include singlepoint-in-time-instruments like the Acute Physiology and Chronic Evaluation (APACHE) [28] and progressive instruments like the Sequential Organ Failure Assessment (SOFA) [29]. However, these tools remain less applicable 
Table 3 In-hospital mortality in prognostic categories of different vital signs

\begin{tabular}{|c|c|c|c|c|}
\hline \multirow[b]{2}{*}{$\underline{\text { Variable }}$} & \multicolumn{2}{|c|}{$\begin{array}{l}\text { Development } \\
\text { cohort }(N=167)\end{array}$} & \multicolumn{2}{|c|}{$\begin{array}{l}\text { Validation cohort } \\
(N=150)\end{array}$} \\
\hline & $\overline{n^{*}}$ & Mortality (\%) & $\bar{n}$ & Mortality (\%) \\
\hline \multicolumn{5}{|l|}{ Temperature $\left({ }^{\circ} \mathrm{C}\right)$} \\
\hline$\leq 36.5$ & 51 & $28 \%$ & 17 & $53 \%$ \\
\hline 36.6 to 38.5 (reference) & 84 & $19 \%$ & 67 & $30 \%$ \\
\hline$\geq 38.6$ & 32 & $28 \%$ & 66 & $24 \%$ \\
\hline \multicolumn{5}{|l|}{$\begin{array}{l}\text { Mean arterial } \\
\text { pressure }(\mathrm{mmHg})\end{array}$} \\
\hline$\leq 69$ & 56 & $30 \%$ & 52 & $39 \%$ \\
\hline 70 to 109 (reference) & 86 & $19 \%$ & 95 & $25 \%$ \\
\hline$\geq 110$ & 13 & $23 \%$ & 3 & $33 \%$ \\
\hline \multicolumn{5}{|l|}{$\begin{array}{l}\text { Respiratory rate } \\
\text { (cycles/minute) }\end{array}$} \\
\hline$<30$ (reference) & 102 & $15 \%$ & 65 & $28 \%$ \\
\hline$\geq 30$ & 65 & $37 \%$ & 85 & $32 \%$ \\
\hline \multicolumn{5}{|l|}{ Pulse rate (beats/minute) } \\
\hline$<100$ (reference) & 61 & $20 \%$ & 16 & $25 \%$ \\
\hline$\geq 100$ & 106 & $26 \%$ & 134 & $31 \%$ \\
\hline \multicolumn{5}{|l|}{ Glasgow coma score } \\
\hline 15 (reference) & 133 & $20 \%$ & 115 & $23 \%$ \\
\hline 14 to 13 & 24 & $33 \%$ & 13 & $39 \%$ \\
\hline$\leq 12$ & 10 & $40 \%$ & 14 & $64 \%$ \\
\hline
\end{tabular}

"Number of patients in category. The table shows mortality in prognostic categories of different vital signs among patients with sepsis hospitalized in Uganda. The individual categories were created according to how vital signs predicted mortality in the development cohort.

in acute care settings, where patients spend limited time. They are even less applicable in resource-limited settings, where support systems like laboratories are weak. Scoring systems like MEWS that use simple, yet treatmentmodifiable variables, are therefore a welcome addition. However, there are not enough data supporting their use in both triage and monitoring. Our results represent one

Table 4 Mortality at different scores of the proposed prognostic index

\begin{tabular}{llllll}
\hline & \multicolumn{2}{l}{ Sevelopment cohort } & & \multicolumn{2}{l}{ Validation cohort } \\
\cline { 2 - 3 } \cline { 5 - 6 } & $\begin{array}{l}\text { Number of } \\
\text { patients }\end{array}$ & Died (\%) & & $\begin{array}{l}\text { Number of } \\
\text { patients }\end{array}$ & Died (\%) \\
\hline $\mathbf{0}$ & 28 & $11 \%$ & 3 & $33 \%$ \\
$\mathbf{1}$ & 32 & $6.3 \%$ & 26 & $27 \%$ \\
$\mathbf{2}$ & 57 & $25 \%$ & 38 & $16 \%$ \\
$\mathbf{3}$ & 36 & $36 \%$ & 59 & $34 \%$ \\
$\mathbf{4}$ & 12 & $42 \%$ & 18 & $33 \%$ \\
$\mathbf{5}$ & 2 & $100 \%$ & 6 & $83 \%$ \\
\hline
\end{tabular}

The table shows the number of patients and proportions that died in hospital at increasing scores of the proposed prognostic index by cohort among patients with sepsis hospitalized in Uganda.
Table 5 Prediction of mortality by the Modified Early Warning Score (MEWS) and the proposed index

\begin{tabular}{|c|c|c|c|c|}
\hline \multirow[b]{2}{*}{ Score } & \multicolumn{2}{|c|}{$\begin{array}{l}\text { Development cohort } \\
(\mathrm{N}=167)\end{array}$} & \multicolumn{2}{|c|}{$\begin{array}{l}\text { Validation cohort } \\
(\mathrm{N}=150)\end{array}$} \\
\hline & OR $(95 \% \mathrm{Cl})$ & $P$ & OR $(95 \% \mathrm{Cl})$ & $P$ \\
\hline \multicolumn{5}{|c|}{ Proposed index } \\
\hline 0 to 2 & Ref & - & Ref & - \\
\hline$\geq 3$ & 3.4 (1.6 to 7.3$)$ & 0.001 & 2.3 (1.1 to 4.7 ) & 0.031 \\
\hline \multicolumn{5}{|l|}{ MEWS } \\
\hline 0 to 4 & Ref & - & Ref & - \\
\hline$\geq 5$ & 2.5 (1.2 to 5.3$)$ & 0.017 & 1.8 (0.74 to 4.2$)$ & 0.204 \\
\hline
\end{tabular}

The table shows unadjusted odds ratios and $95 \%$ confidence intervals of in-hospital death at scores $\geq 5$ for MEWS and at scores $\geq 3$ for the proposed index among patients with sepsis hospitalized in Uganda. OR, odds ratio; $\mathrm{Cl}$, confidence interval; MEWS, Modified Early Warning Score.

approach to creating newer/simpler triage/monitoring systems.

Monitoring vital signs in acutely ill patients can also aid integrated management of acute and chronic illnesses. For example, in our data, patients with high BP at admission had higher mortality than those with normal BP. As hypertension in sepsis is not usually associated with high mortality [30], this finding may suggest treatment opportunities that could be addressed by better vital signs monitoring, for example being careful with fluid administration in patients with pre-existing hypertension, while still giving appropriate fluid resuscitation to those who may have elevated BP at admission but without pre-existing hypertension.

Our findings suggest that studies using vital signs data to monitor outcomes should consider modeling these data more flexibly, yet efficiently. Previous studies have reported relationships between BP and mortality in binary fashion, for example mortality at MAP $<60 \mathrm{mmHg}$ versus $\geq 60 \mathrm{mmHg}$, or in incremental categories, for example mortality per 10 unit increase in BP starting from a reference category $[8,31]$. We suggest that future studies consider the approximately U-shaped patterns in the relationships of mortality with both BP and temperature and the roughly linear patterns for respiratory and pulse rates. These patterns should be considered when measuring vital signs at one point in time, for example at admission, or progressively, for example at post-admission intervals [32].

Our findings have some limitations. As vital signs were infrequently measured, we were not able to use multiple measurements for each vital sign, in addition to imputing some missing values in the development cohort, which may affect the precision of our measurements. Restricted cubic splines are a flexible way of assessing relationships. However, the resulting curves are smoothed, and, especially at the extremes, where data are usually thin, the predictions can be unreliable. As the sample sizes 
were small, the OR estimates, especially in the validation cohort, had wide $95 \%$ CIs and, for the estimates assessing the performance of MEWS, a $P$ value not reaching statistical significance. Also, binary modeling, which gives an equal score at all GCS levels, may lead to loss of information. Future studies should examine more efficient, yet still simple, ways of modeling not only GCS, but also other vital signs. Despite these limitations, our findings have potential to guide the development of prognostic indexes incorporating vital signs data that can be used to monitor treatment. We included in the proposed index only treatment-modifiable variables, and, despite the small sample sizes, prediction patterns in the development cohort were replicated in the validation cohort.

\section{Conclusions}

Among patients hospitalized with sepsis in sub-Saharan Africa, a prognostic index incorporating vital signs data with less segmentation in the values of included variables and giving no score for low values of respiratory and pulse rates adequately predicted mortality. Such an index may be easier to implement than existing related indexes when triaging acutely-ill patients. In addition, future studies using a similar approach on post-admission vital signs may develop prognostic indexes that can be used to monitor treatment among acutely-ill patients, especially in resource-limited settings.

\section{Key messages}

- Existing prognostic indexes that use vital signs information incorporate this information in ways that are not always efficient

- We used restricted cubic splines to assess how admission vital signs data predict mortality in patients with sepsis

- Guided by these predictions, we incorporated treatment-modifiable routine vital signs into a prognostic index with reduced segmentation in the values of incorporated variables

- The resulting prognostic index adequately predicted mortality in two cohorts of patients with sepsis

- Future studies using a similar approach on post-admission vital signs data could develop indexes that can be used to monitor treatment, especially in resource-limited settings

\footnotetext{
Abbreviations

BP: blood pressure; Cl: confidence interval; CVP: central venous pressure: DBP: diastolic blood pressure; GCS: Glasgow coma score; HOTEL: hypotension, oxygen saturation, temperature, electrocardiographic abnormality, and loss of independence; IQR: inter-quartile range; IRC: Institutional Review Committee; MAP: mean arterial pressure; MEWS: Modified Early Warning Score; MI: multiple imputations; OR: odds ratio; SBP: systolic blood pressure; SIRS: systemic inflammatory response syndrome; SSA: sub-Saharan Africa; ScvO2: central venous oxygen saturation;
}

TOTAL: tachypnea, oxygen saturation, temperature, alertness, and loss of independence.

\section{Competing interests}

The authors declare that they have no competing interests.

\section{Authors' contributions}

SBA conceptualized the study, prepared the data, performed the analyses, and wrote and edited the manuscript. AA conceptualized the study, and wrote and edited the manuscript. SR conceptualized the study, prepared the data, and wrote and edited the manuscript. All authors read and approved the final version of manuscript.

\section{Acknowledgements}

Funding for data collection for the source cohort studies was provided by the University of Virginia, Charlottesville, Center for Global Health - Pfizer Initiative in International Health.

\section{Author details}

'Department of Medicine, Mbarara Regional Referral Hospital, P.O. Box 40, Mbarara, Uganda. ${ }^{2}$ Department of Medicine, Mbarara University of Science and Technology, P.O. Box 1410, Mbarara, Uganda. ${ }^{3}$ Department of Epidemiology and Biostatistics, University of California San Francisco, 550 16th Street, Second Floor, Mission Hall: Global Health and Clinical Sciences Building, San Francisco, CA 94158-2549, USA. ${ }^{4}$ Department of Medicine, College of Health Sciences, Makerere University, P.O. Box 7072, Kampala, Uganda.

Received: 21 November 2014 Accepted: 19 February 2015

Published online: 16 March 2015

\section{References}

1. Jacob ST, Moore CC, Banura P, Pinkerton R, Meya D, Opendi P, et al. Severe sepsis in two Ugandan hospitals: a prospective observational study of management and outcomes in a predominantly HIV-1 infected population. PLoS One. 2009;: :e7782.

2. Andrews B, Muchemwa L, Kelly P, Lakhi S, Heimburger DC, Bernard GR. Simplified severe sepsis protocol: a randomized controlled trial of modified early goal-directed therapy in Zambia. Crit Care Med. 2014;42:2315-24.

3. Dellinger RP, Levy MM, Rhodes A, Annane D, Gerlach H, Opal SM, et al. Surviving sepsis campaign: international guidelines for management of severe sepsis and septic shock: 2012. Crit Care Med. 2013;41:580-637.

4. Rivers E, Nguyen B, Havstad S, Ressler J, Muzzin A, Knoblich B, et al. Early goal-directed therapy in the treatment of severe sepsis and septic shock. N Engl J Med. 2001;345:1368-77.

5. Hilton AK, Bellomo R. A critique of fluid bolus resuscitation in severe sepsis. Crit Care. 2012;16:302.

6. Pro Cl, Yealy DM, Kellum JA, Huang DT, Barnato AE, Weissfeld LA, et al. A randomized trial of protocol-based care for early septic shock. N Engl J Med. 2014;370:1683-93.

7. Kwizera A, Dunser M, Nakibuuka J. National intensive care unit bed capacity and ICU patient characteristics in a low income country. BMC Res Notes. 2012;5:475.

8. Ssekitoleko R, Pinkerton R, Muhindo R, Bhagani S, Moore CC. Aggregate evaluable organ dysfunction predicts in-hospital mortality from sepsis in Uganda. Am J Trop Med Hyg. 2011;85:697-702.

9. Opio MO, Nansubuga G, Kellett J. Validation of the VitalPAC Early Warning Score (ViEWS) in acutely ill medical patients attending a resource-poor hospital in sub-Saharan Africa. Resuscitation. 2013;84:743-6.

10. Corfield AR, Lees F, Zealley I, Houston G, Dickie S, Ward K, et al. Utility of a single early warning score in patients with sepsis in the emergency department. Emerg Med J. 2014;31:482-7.

11. Kyriacos U, Jelsma J, Jordan S. Monitoring vital signs using early warning scoring systems: a review of the literature. J Nurs Manag. 2011;19:311-30

12. Burch VC, Tarr G, Morroni C. Modified early warning score predicts the need for hospital admission and inhospital mortality. Emerg Med J. 2008;25:674-8.

13. Griffiths JR, Kidney EM. Current use of early warning scores in UK emergency departments. Emerg Med J. 2012;29:65-6.

14. Wheeler I, Price C, Sitch A, Banda P, Kellett J, Nyirenda M, et al. Early warning scores generated in developed healthcare settings are not sufficient at predicting early mortality in Blantyre, Malawi: a prospective cohort study. PLoS One. 2013;8:e59830. 
15. Opio MO, Nansubuga G, Kellett J. In-hospital mortality of acutely ill medical patients admitted to a resource poor hospital in sub-Saharan Africa and to a Canadian regional hospital compared using the abbreviated VitaIPAC Early Warning Score. Eur J Intern Med. 2014;25:142-6.

16. Mohammed M, Hayton R, Clements G, Smith G, Prytherch D. Improving accuracy and efficiency of early warning scores in acute care. Br J Nurs. 2009;18:18-24.

17. Kyriacos $U$, Jelsma J, James M, Jordan S. Monitoring vital signs: development of a modified early warning scoring (MEWS) system for general wards in a developing country. PLoS One. 2014;9:e87073.

18. Subbe CP, Kruger M, Rutherford P, Gemmel L. Validation of a modified Early Warning Score in medical admissions. QJM. 2001;94:521-6.

19. Asiimwe SB, Muzoora C, Wilson LA, Moore CC. Bedside measures of malnutrition and association with mortality in hospitalized adults. Clin Nutr. 2014; in press.

20. Dellinger RP, Levy MM, Rhodes A, Annane D, Gerlach H, Opal SM, et al. Surviving Sepsis Campaign: international guidelines for management of severe sepsis and septic shock, 2012. Intensive Care Med. 2013;39:165-228.

21. Meaney E, Alva F, Moguel R, Meaney A, Alva J, Webel R. Formula and nomogram for the sphygmomanometric calculation of the mean arterial pressure. Heart. 2000;84:64.

22. Gerdin M, Roy N, Khajanchi M, Kumar V, Dharap S, Fellander-Tsai L, et al. Predicting early mortality in adult trauma patients admitted to three public university hospitals in urban India: a prospective multicentre cohort study. PLoS One. 2014;9:e105606.

23. Durrleman S, Simon R. Flexible regression models with cubic splines. Stat Med. 1989:8:551-61.

24. Patrick R, Willi S. Multivariable modeling with cubic regression splines: A principled approach. Stat J. 2007;7:45-70.

25. Vonta F, Karagrigoriou A. Variable selection strategies in survival models with multiple imputations. Lifetime Data Anal. 2007;13:295-315.

26. Guex N, Migliavacca E, Xenarios I. Multiple imputations applied to the DREAM3 phosphoproteomics challenge: a winning strategy. PLoS One. 2010;5:e8012.

27. Harrell Jr FE, Lee KL, Mark DB. Multivariable prognostic models: issues in developing models, evaluating assumptions and adequacy, and measuring and reducing errors. Stat Med. 1996;15:361-87.

28. Wong DT, Knaus WA. Predicting outcome in critical care: the current status of the APACHE prognostic scoring system. Can J Anaesth. 1991;38:374-83.

29. Jones AE, Trzeciak S, Kline JA. The Sequential Organ Failure Assessment score for predicting outcome in patients with severe sepsis and evidence of hypoperfusion at the time of emergency department presentation. Crit Care Med. 2009:37:1649-54.

30. Nunes JP. Arterial hypertension and sepsis. Rev Port Cardiol. 2003;22:1375-9.

31. Jacob ST, Banura P, Baeten JM, Moore CC, Meya D, Nakiyingi L, et al. The impact of early monitored management on survival in hospitalized adult Ugandan patients with severe sepsis: a prospective intervention study. Crit Care Med. 2012;40:2050-8.

32. Ghassemi M, Naumann T, Doshi-Velez F, Brimmer N, Joshi R, Rumshisky A, et al. Unfolding physiological state: mortality modelling in intensive care units. KDD: Proc Int Con Knowl Discov Data Mining. 2014;2014:75-84.

\section{Submit your next manuscript to BioMed Central and take full advantage of:}

- Convenient online submission

- Thorough peer review

- No space constraints or color figure charges

- Immediate publication on acceptance

- Inclusion in PubMed, CAS, Scopus and Google Scholar

- Research which is freely available for redistribution 